\title{
CELL-SELEX: Novel Perspectives of Aptamer-Based Therapeutics
}

\author{
Ke-Tai Guo ${ }^{1}$, Angela Paul ${ }^{1}$, Christian Schichor ${ }^{2}$, Gerhard Ziemer ${ }^{1}$ and Hans P. Wendel ${ }^{1, *}$ \\ 1 Department of Thoracic, Cardiac and Vascular surgery, University Hospital Tuebingen, \\ Calwerstr.7/1, D72076 Tuebingen, Germany \\ 2 Department of Neurosurgery, Ludwig-Maximilians-University, Klinikum Grosshadern, \\ Marchioninistr. 15, D81377 Munich, Germany \\ * Author to whom correspondence should be addressed; E-mail: hp.wendel@uni-tuebingen.de
}

Received: 18 December 2008; in revised form: 3 April 2008 / Accepted: 24 April 2008 /

Published: 24 April 2008

\begin{abstract}
Aptamers, single stranded DNA or RNA molecules, generated by a method called SELEX (systematic evolution of ligands by exponential enrichment) have been widely used in various biomedical applications. The newly developed Cell-SELEX (cell based-SELEX) targeting whole living cells has raised great expectations for cancer biology, -therapy and regenerative medicine. Combining nanobiotechnology with aptamers, this technology opens the way to more sophisticated applications in molecular diagnosis. This paper gives a review of recent developments in SELEX technologies and new applications of aptamers.
\end{abstract}

Keywords: cell-SELEX, aptamer, nanotechnology

\section{Introduction}

SELEX (systematic evolution of ligands by exponential enrichment) is a method to generate DNA or RNA ligands from a combinatorial library. This oligonucleotide library consists of single stranded modified or unmodified RNA or DNA. The ligands that emerge from SELEX have been called aptamers [1]. Aptamers have promising advantages compared to antibodies: they can be produced easily and inexpensively and are simple to chemically modify and integrate into different analytical schemes. Aptamers can retain their binding and inhibitory behavior after immobilization on a carrier material [2] or after delivery into animals [3] and can be labeled with various functional groups [4]. 
These properties of aptamers have led to their application in many areas of biomedical sciences such as purification processes [5], target validation [6], drug development [7], diagnostics [8], MRI-based cell tracking [9] and even therapy [3].

\section{SELEX methodology and clinical applications of aptamers}

The first SELEX experiments were performed in the 1990s by the independent research groups of Gold and Ellington [1, 10-11], proceeding typically as follows: First a combinatorial nucleic acid library (DNA or RNA) is synthesized. The sequence of the library is composed of random sequences in the middle and flanked by fixed sequences as primer binding sites. The length of the random region is normally between 20 to $40 \mathrm{bp}$, which creates a library with a large number of random sequences $\left(10^{15}\right.$ to $\left.10^{16}\right)$. The size of the library is large enough to saturate the relevant sequence space. The library is then incubated with the desired target molecule under conditions suitable for binding. Next, the unbound nucleic acids are partitioned from those bound specifically to the target molecule, which are then eluted from the target molecule and amplified by PCR. This selection procedure is reiterated for several rounds until the resulting sequences are highly enriched. The selected nucleic acids are subjected to DNA sequencing and screened for potential binding affinity. SELEX technology generates aptamers with high binding affinity and specificity. Their advantages have made them very promising in analytical, diagnostic and therapeutic applications [12-15].

As small molecules, with a half-life of minutes to hours due to nuclease degradation, aptamers can be rapidly cleaned from the bloodstream by the kidneys. The applications of unmodified aptamers are mainly focused on treating transient conditions such as blood clotting [16-17], or treating organs, for example the eye where local delivery is possible [18-19]. This rapid clearance is an advantage in some applications such as in vivo diagnostic imaging, for example a tenascin-binding aptamer under development for cancer imaging [20]. There are several modifications available, such as 2'-fluorinesubstituted pyrimidines, polyethylene glycol (PEG) linkage, etc. (both of which are used in Macugen, an FDA-approved aptamer), that can increase the half-life of aptamers easily to a day or even a week [21-22].

The first aptamer approved for use in man is a RNA-based molecule (Macugen, Pegaptanib) that is administered locally to treat age-related macular degeneration by targeting vascular endothelial growth factor (VEGF). Pegaptanib is a pegylated anti-VEGF aptamer, a single stranded nucleic acid that binds with high specificity to a particular target. Pegaptanib specifically binds to VEGF165, a protein that plays a critical role in angiogenesis (the formation of new blood vessels) and in increased permeability (leakage from blood vessels), two of the primary pathological processes responsible for the vision loss associated with AMD (age-related macular degeneration). The brand name Macugen for Pegaptanib denotes an anti-angiogenic medicine for the treatment of neovascular (wet) AMD. FDA-approval was granted in December 2004 [23-24].

The most advanced aptamer in the cancer setting is AS1411, formerly known as AGRO100, which is being administered systemically in clinical trials [25]. AS1411 is a 26-mer unmodified guanosine-rich oligonucleotide, which induces growth inhibition in vitro, and has shown activity against human tumor xenografts in vivo. The mechanism underlying its antiproliferative effects in cancer cells seems to involve initial binding to cell surface nucleolin and internalization, leading to an inhibition of DNA 
replication. In contrast to other unmodified oligonucleotides, AS1411 is relatively stable in serumcontaining medium through the formation of dimers and a quartet structure. In a dose escalation phase I study in patients with advanced solid tumors, doses up to $10 \mathrm{mg} / \mathrm{kg} / \mathrm{d}$ (using a four or seven continuous infusion regime) have been tested [26]. Promising signs of activity were reported (multiple cases of stable disease and one near complete response in a patient with renal cancer) in the absence of any significant adverse effects. Further trials are ongoing in renal and non-small cell lung cancers [27]., Additional aptamers have been described in preclinical studies against several cancer targets, such as the antiapoptotic, the transcription factor signal transducer and activator of transcription 3 , and the $\mathrm{Ku}$ proteins [28]. Apoptosis is an essential cellular process critical for tissue homeostasis and animal development in metazoans. Abnormal inactivation of apoptosis can result in uncontrolled cell growth, leading to development of cancer and autoimmune disorders. The human proto-oncogene bcl2 controls apoptosis by an evolutionarily conserved cell death pathway. Members of this family can be either antiapoptotic or proapoptotic and can form heterodimers with other selected family members to affect apoptosis. CED-9 is a cell death inhibitor and a homologue of human Bcl-2. Developing effective therapeutic methods to correct or reverse inappropriate apoptosis is a critical issue in clinical medicine. Aptamers have been successfully generated to antagonize the prosurvival activity of CED-9 and kill cells through the normally programmed cell death pathway. These results suggest that RNA aptamers for key cell death regulators can yield, or serve as leads, to generate potent compounds that modulate apoptosis in vivo [29-31]. The transcription factor signal transducer and activator of transcription (Stat3) plays an essential role in embryonic development and assumes specialized tasks in many differentiated tissues. Constitutively activated Stat3 has been found in tumor cell lines and primary tumors and plays a crucial role in tumor cell survival and proliferation [32]. The peptide aptamers that have been generated to inhibit the oncogenic action of Stat3 in tumor cells, can recognize the Stat3 DNA binding domain and inhibit DNA binding and transactivation by Stat3 following EGF stimulation of cells. Inhibiting the Stat 3 functions through interaction with peptide aptamers counteracts the transformed phenotype and could become useful in targeted tumor therapy [33].

\subsection{Cell-SELEX}

A modification of the traditional SELEX process that uses whole living cells as target was named cell-SELEX (Figure 1). Aptamers with high affinity and specificity for cells have been produced successfully, demonstrating that complex targets, including tumor cells and tissues, are compatible with the SELEX process [34]. Using purified proteins as targets has the obvious advantage of easy control to achieve optimal enrichment during the selection process. But Cell-SELEX is favored in some special cases, when the clear marker target is unknown. Furthermore, since the target protein domain may be shielded and inaccessible on a cell surface, it reflects a more physiological condition when the protein is displayed on the cell surface rather than isolated as purified protein., 


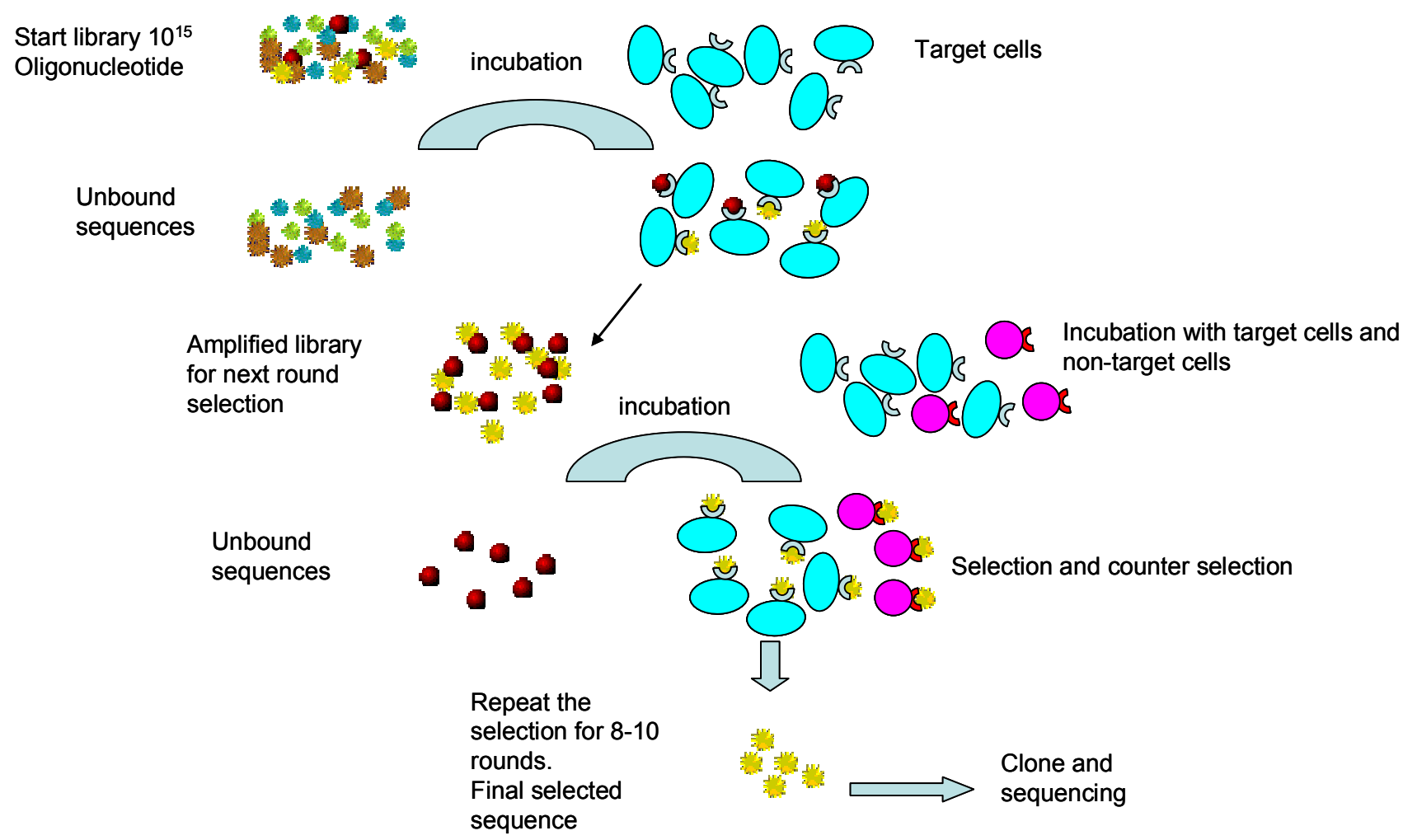

Figure 1. Cell-SELEX procedure. In the Cell-SELEX procedure, the target for SELEX is whole living cells. In this case, aptamers bind with the membrane protein of the cells. The selection of aptamer is cyclically repeating, during selection rounds the binding affinity can be controlled by Flow cytometry assay to decide the end of selection.

The whole cell selection process is easy to handle, fast and reproducible. It makes aptamers an effective tool for molecular medicine and biomarker discovery. In recent reports, the Cell-SELEX technology plays an important role in cancer biology. For most cancer cells, there is always a shortage of high specific surface markers for diagnosis and therapy. Aptamers generated from whole living cells are the optimal molecular probe to characterize target cells on a molecular level. When bound with the membrane receptors of the cell lines, they provide an effective approach for the discovery of biomarkers as disease signals. The development of aptamer probes for molecular signatures on the cancer cell surface is highly specific, allows defining of tumors, and creates an opportunity for more 'personalized' treatment. The generation of aptamers to normal cells and cancer cells enables a comparative strategy to identify differences at a molecular level and to promote the discovery of molecular features of cancer cells.

The advantages of whole living Cell-SELEX-based aptamers facilitate the development of and simplify the search for molecular probes for diseased cell recognition [35-37]. Aptamers can also be used for the recognition of differences at a molecular level between any two given populations of cells, such as tumor cell type 1 vs. type 2 . These differences possess great significance in aiding the understanding of the biological processes and mechanisms of diseases. It is difficult to identify the molecular differences and validate them specifically with current technology. Tang et al. [38] developed aptamers for the specific recognition of Burkitt's lymphoma cells. Burkitt's lymphoma, an acute blood cell cancer, is one of the most progressive human cancers. Early diagnosis and targeted therapy are crucial for patient treatment. However, the lack of effective molecular probes to recognize 
cell surface biomarkers prevents early diagnosis of Burkitt's lymphoma and makes the study of its developing mechanism difficult if not impossible. Of the many possible probes, aptamers hold the greatest potential in molecular recognition and targeting. The generated aptamers showed excellent specificity and strong affinity for Ramos cells, a Burkitt's lymphoma cell line. The selected probes are very helpful for understanding the molecular interactions and recognition events on Ramos cells, as well as for the future medical diagnosis and treatment of lymphoma. Tumor Cell-SELEX also demonstrates the ability of aptamers to identify known tumor markers, such as tenascin-C [21]. Tenascin- $\mathrm{C}$ is an extracellular matrix protein that is overexpressed during tissue remodeling processes, including tumor growth. It is believed to be a stromal marker for epithelial malignancy [39]. TTA1, an aptamer against the extracellular matrix protein tenascin- $\mathrm{C}$, can be taken up by a variety of solid tumors including breast, glioblastoma, lung, and colon. Rapid uptake by tumors and fast clearance from the blood and other non-target tissues enables clear tumor imaging. This aptamer shows high potential for in vivo delivery of radioisotopes or cytotoxic agents [20]. Cerchia et al. adopted a wholecell-SELEX strategy to target RET receptor (rearranged during transfection) in a complex environment that was expected to expose a native protein to the selection procedure, thus best mimicking in vivo conditions. Nuclease-resistant aptamers, that can recognize the human receptor tyrosine kinase RET, were obtained using RET-expressing cells as targets in a modified SELEX procedure [40]. The aptamers also blocked RET downstream signaling and subsequent molecular and cellular events. The fact that aptamers with anti-oncogenic activity were isolated in the absence of a specific selective pressure, suggests that the method could be used to identify active macromolecules with high potential therapeutic interest against other transmembrane receptors. Guo et al. generated aptamers for the isolation of mesenchymal stem cells from whole bone marrow [41]. The isolated MSCs can be used directly for local injection or other applications. When bound to magnetic particles aptamers could be used for stem cell tracking [9]. Furthermore, they found, after immobilizing aptamers against osteoblasts on titanium [42], that aptamer-coated titanium surfaces can enrich osteoblasts quickly and efficiently. This fast and highly efficient method based on aptamers will bring novel aspects of MSCs to the field of regenerative medicine and tissue engineering.

\section{Aptamers and nanobiotechnology}

The term 'nanotechnology' is defined as the processing of separation, consolidation, and deformation of materials by one atom or one molecule, and includes the understanding and control of matter at dimensions of $1-100 \mathrm{~nm}$ where unique phenomena enable novel applications. The bulk physical properties of materials often become very different when broken down at nano-scale; therefore, nanotechnology generates new opportunities and applications. Nanomaterials and devices provide unique opportunities to advance medicine referred to as 'nanomedicine', which impact diagnosis, monitoring and treatment of diseases as well as control and understanding of biological systems [43]. Applying nanotechnology to the aptamer field, Tan et al. have successfully constructed an aptamer-conjugated nanoparticle for the collection and detection of multiple cancer cells [44]. They attached aptamers to magnetic nanoparticles (MNP) and fluorescent nanoparticles (FNP) to develop a specific platform for collecting and imaging intact leukemia cells from mixed cell- and whole blood samples. Furthermore, they developed an aptamer-conjugated nanoparticles protocol to perform the 
extraction of multiple cancer cell targets using additional high-affinity aptamers for recognition. They utilized $65 \mathrm{~nm}$ silica-coated MNP instead of the traditional micrometer-sized magnetic polymer beads. The small size and relatively high surface area of NPs provide enhanced extraction capabilities and eliminate the need for pre-sample cleanup. Upon excitation, the FNP conjugated aptamer can produce significantly higher signal intensity and -stability than other individual dye probes. The method of conjugating aptamers with nanoparticles has the potential to be used in profiling applications with furhter development of aptamer technology [45].

Another new application is to fabricate electrochemical aptamers as molecular recognition elements for nanoscale biosensors. Taking advantage of small size aptamers from 10-60 bp in length, the binding with target molecules can occur inside the electrical double layer and in saline solution. By using aptamers for recognition, no labeling is required for electrochemical sensors, and signal-on architecture that is only possible with aptamers, has improved their sensitivity. The sensors can be recycled because of the reversibility of aptamer configurations. Alternatively, bound proteins may simply be washed off without damaging the aptamers. Aptamers have already proven to be superior to antibodies in nanosensors [46-49].

Aptamer-conjugated nanoparticles for drug delivery to treat cancer cells come up as another new and exciting application. One of the biggest issues with current cancer therapies is that most of them kill both healthy and cancerous cells. Drug delivery specialists are now presented with the challenge and the opportunity to increase the therapeutic index and ultimately deliver targeted therapies to cancer cells while minimizing off-target side effects. These bioconjugates represent an exciting prospect in the advancing field of cancer nanotechonology and hold significant promise for future cancer treatment. By modifying the controlled-release polymer system or tweaking the aptamer targeting group it may be possible to produce a diverse range of specific and selective bioconjugates (Figure 2). In this way, drug delivery 'vehicles' could target numerous significant human cancers. The application of nanotechnology to cancer therapy is expected to result in future therapeutic modalities that are superior to our current approach. Most importantly, this is no longer farfetched science: nanoscale drug delivery vehicles are already getting closer to clinical realization.

\section{Aptamers and gene silencing}

When conjugated to aptamers, the mediate targeted delivery of small interfering RNAs (siRNAs) can be greatly improved resulting in higher therapeutic efficacy and safety [50-51]. This approach has the advantage that therapeutics can be composed entirely of RNA. The aptamer-siRNA chimeric RNAs are capable of cell type-specific binding and delivery of functional siRNAs into cells [52]. For example, the aptamer portion of the chimeras mediates binding to PSMA (prostate-specific membrane antigen), a cell-surface receptor overexpressed in prostate cancer cells and tumor vascular endothelium, whereas the siRNA portion targets the expression of survival genes. When applied to cells expressing PSMA, these RNAs are internalized and processed by Dicer, resulting in depletion of the siRNA target proteins and cell death. In contrast, the chimeras do not bind to or function in cells that do not express PSMA. These reagents also specifically inhibit tumor growth and mediate tumor regression in a xenograft model of prostate cancer [53]. These studies demonstrate a novel approach for targeted delivery of siRNAs with numerous potential applications, including cancer therapeutics. 
Short aptamers (25-35bp) have been generated that bind to a wide variety of targets with a high affinity. It should be possible to design siRNA-aptamer chimeras with longer strands (45-55bp) that are within the range of technical and commercial feasibility for synthesis, however, molecules longer than that may be difficult to synthesize on a commercial scale. Although short aptamer-conjugates work for local delivery in vivo, they are rapidly cleared by the kidney and have a half-life too short for systemic therapy unless they are incorporated into larger particles.

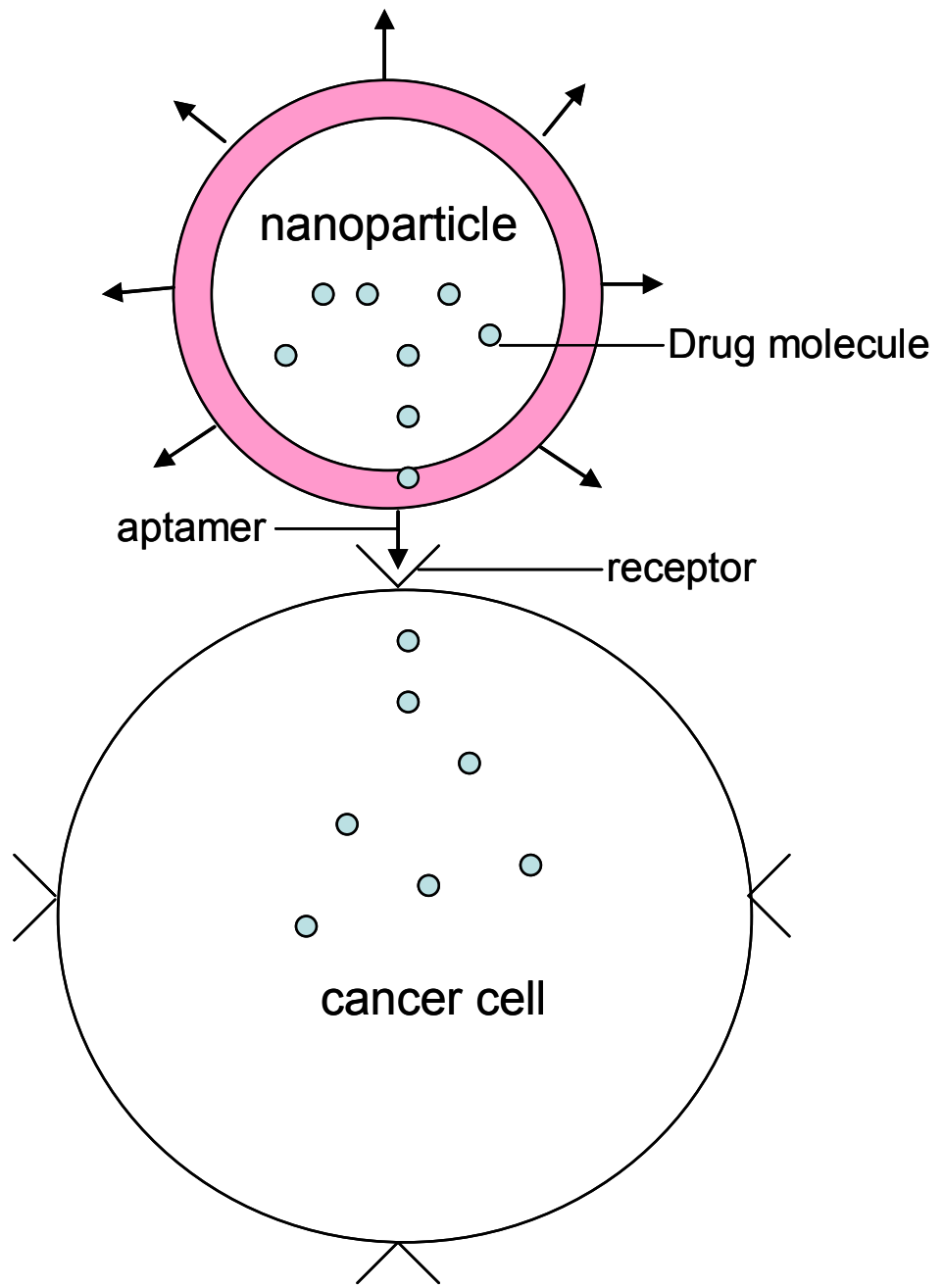

Figure 2. Apatamer-conjugated nanoparticle for delivery of drugs to treat cancer. The aptamer-conjugated nanoparticle acts as a drug delivery 'vehicle', first the aptamer finds the cancer cell and binds it, then the nanoparticle can release the drug molecules to kill the cancer cell.

\section{Conclusion}

The encouraging results obtained by aptamers based on their intrinsic properties and the versatility of the SELEX procedure generates new applications in diagnostic as well as therapeutic fields. The ability to discriminate between two closely related targets, for example a cancerous cell and an untransformed cell of the same tissue type, makes aptamers suitable as imaging reagents for noninvasive diagnostic procedures. Even though the combined use of aptamers with nanoparticles is still a 
novel concept, a growing number of valuable results have been reported contributing to cancer medicine and molecular medicine and opening up a huge potential for numerous clinical applications.

\section{References and Notes}

1. Tuerk, C.; Gold, L. Systematic evolution of ligands by exponential enrichment: RNA ligands to bacteriophage T4 DNA polymerase. Science. 1990, 249, 505-510.

2. Farokhzad, O.C.; Jon, S.; Khademhosseini, A.; Tran, T.N.; Lavan, D.A.; Langer, R. Nanoparticleaptamer bioconjugates: a new approach for targeting prostate cancer cells. Cancer Res. 2004, 64, 7668-7672.

3. Nimjee, S.M.; Rusconi, C.P.; Sullenger, B.A. Aptamers: an emerging class of therapeutics. Annu. Rev. Med. 2005, 5, 555-583.

4. Farokhzad, O.C.; Karp, J.M.; Langer, R. Nanoparticle-aptamer bioconjugates for cancer targeting. Expert Opin. Drug Deliv. 2006, 3, 311-324.

5. Romig, T.S.; Bell, C.; Drolet, D.W. Aptamer affinity chromatography: combinatorial chemistry applied to protein purification. J. Chromatogr. B. Biomed. Sci. Appl. 1999, 731, 275-284.

6. Burgstaller, P.; Girod, A.; Blind, M. Aptamers as tools for target prioritization and lead identification. Drug Discov. Today. 2002, 7, 1221-1228.

7. Green, L.S.; Bell, C.; Janjic, N. Aptamers as reagents for high-throughput screening. Biotechniques. 2001, 30, 1094-1096.

8. Brody, E.N.; Willis, M.C.; Smith, J.D.; Jayasena, S.; Zichi, D.; Gold, L. The use of aptamers in large arrays for molecular diagnostics. Mol. Diagn. 1999, 4, 381-388.

9. Schafer, R.; Wiskirchen, J.; Guo, K.; Neumann, B.; Kehlbach, R.; Pintaske, J.; Voth, V.; Walker, T.; Scheule, A.M.; Greiner, T.O.; Hermanutz-Klein, U.; Claussen, C.D.; Northoff, H.; Ziemer, G.; Wendel, H.P. Aptamer-based isolation and subsequent imaging of mesenchymal stem cells in ischemic myocard by magnetic resonance imaging. Rofo. 2007, 179, 1009-1015.

10. Ellington, A.D.; Szostak, J.W. In vitro selection of RNA molecules that bind specific ligands. Nature. 1990, 346, 818-822.

11. Ellington, A.D.; Szostak, J.W. Selection in vitro of single-stranded DNA molecules that fold into specific ligand-binding structures. Nature. 1992, 355, 850-852.

12. Djordjevic, M. SELEX experiments: new prospects, applications and data analysis in inferring regulatory pathways. Biomol. Eng. 2007, 24, 179-189.

13. Jayasena, S.D. Aptamers: an emerging class of molecules that rival antibodies in diagnostics. Clin. Chem. 1999, 45, 1628-1650.

14. Tombelli, S.; Minunni, M.; Mascini, M. Analytical applications of aptamers. Biosens. Bioelectron. 2005, 15, 20, 2424-2434.

15. Fichou, Y.; Férec, C. The potential of oligonucleotides for therapeutic applications. Trends Biotechnol. 2006, 24, 563-570.

16. Nimjee, S.M.; Rusconi, C.P.; Harrington, R.A.; Sullenger, B.A. The potential of aptamers as anticoagulants. Trends Cardiovasc. Med. 2005, 15, 41-45. 
17. Rusconi, C.P.; Roberts, J.D.; Pitoc, G.A.; Nimjee, S.M.; White, R.R.; Quick, G.Jr.; Scardino, E.; Fay, W.P.; Sullenger, B.A. Antidote-mediated control of an anticoagulant aptamer in vivo. Nat. Biotechnol. 2004, 22, 1423-1428.

18. Quiram, P.A.; Hassan, T.S.; Williams, G.A. Treatment of naïve lesions in neovascular age-related macular degeneration with pegaptanib. Retina. 2007, 27, 851-856.

19. Ehlers, J.P.; Fintak, D.R.; Gupta, O.P.; Regillo, C.D.; Fineman, M.S.; Ho, A.C. Pegaptanib for choroidal neovascularization in treatment-naïve exudative age-related macular degeneration. Ophthalmic Surg. Lasers Imaging. 2007, 38, 371-377.

20. Hicke, B.J.; Stephens, A.W.; Gould, T.; Chang, Y.F.; Lynott, C.K.; Heil, J.; Borkowski, S.; Hilger, C.S.; Cook, G.; Warren, S.; Schmidt, P.G. Tumor targeting by an aptamer. J. Nucl. Med. 2006, 47, 668-678.

21. Daniels, D.A.; Chen, H.; Hicke, B.J.; Swiderek, K.M.; Gold, L. A tenascin-C aptamer identified by tumor cell SELEX: systematic evolution of ligands by exponential enrichment. Proc. Natl. Acad. Sci. USA. 2003, 100, 15416-15421.

22. Boomer, R.M.; Lewis, S.D.; Healy, J.M.; Kurz, M.; Wilson, C.; McCauley, T.G. Conjugation to polyethylene glycol polymer promotes aptamer biodistribution to healthy and inflamed tissues. Oligonucleotides. 2005, 15, 183-195.

23. Ng, E.W.; Shima, D.T,.; Calias, P.; Cunningham, E.T Jr.; Guyer, D.R.; Adamis, A.P. Pegaptanib, a targeted anti-VEGF aptamer for ocular vascular disease. Nat. Rev. Drug Discov. 2006, 5, 123 132.

24. Rakic, J.M.; Blaise, P.; Foidart, J.M. Pegaptanib and age-related macular degeneration. N. Engl. J. Med. 2005, 352, 1720-1721.

25. Teng, Y.; Girvan, A.C.; Casson, L.K.; Pierce, WM.Jr.; Qian, M.; Thomas, S.D.; Bates, P.J. AS1411 alters the localization of a complex containing protein arginine methyltransferase 5 and nucleolin. Cancer Res. 2007, 67, 10491-10500.

26. Ireson, C.R.; Kelland, L.R. Discovery and development of anticancer aptamers. Mol. Cancer Ther. 2006, 5, 2957-2962.

27. Laber, D.A.; Taft, B.S.; Kloecker, G.H.; Bates, P.J.; Trent, J.O.; Miller, D.M. A phase II pilot trial with RP101 in advanced pancreatic carcinoma. Journal of Clinical Oncology, 2006 ASCO Annual Meeting Proceedings Part I, 24, 18S.

28. Zhang, L.;Yoo, S.; Dritschilo, A.; Belyaev, I.; Soldatenkov, V. Targeting Ku protein for sensitizing of breast cancer cells to DNA-damage. Int. J. Mol. Med. 2004, 14, 153-159.

29. Yang, C.; Yan, N.; Parish, J.; Wang, X.; Shi, Y.; Xue, D. RNA aptamers targeting the cell death inhibitor CED-9 induce cell killing in Caenorhabditis elegans. J. Biol. Chem. 2006, 281, 91379144.

30. Horvitz, H.R. Nobel lecture. Worms, life and death. Biosci. Rep. 2003, 23, 239-303.

31. Thompson, C.B. Apoptosis in the pathogenesis and treatment of disease. Science. 1995, 267, 1456-1462.

32. Buerger, C.; Nagel-Wolfrum, K.; Kunz, C.; Wittig, I.; Butz, K.; Hoppe-Seyler, F.; Groner, B. Sequence-specific peptide aptamers, interacting with the intracellular domain of the epidermal growth factor receptor, interfere with Stat3 activation and inhibit the growth of tumor cells. $J$. Biol. Chem. 2003, 278, 37610-37621. 
33. Nagel-Wolfrum, K.; Buerger, C.; Wittig, I.; Butz, K.; Hoppe-Seyler, F.; Groner, B. The interaction of specific peptide aptamers with the DNA binding domain and the dimerization domain of the transcription factor Stat3 inhibits transactivation and induces apoptosis in tumor cells. Mol. Cancer Res. 2004, 2, 170-182.

34. Morris, K.N.; Jensen, K.B.; Julin, C.M.; Weil, M.; Gold, L. High affinity ligands from in vitro selection: complex targets. Proc. Natl. Acad. Sci. USA. 1998, 95, 2902-2907.

35. Shangguan, D.; Li, Y.; Tang, Z.; Cao, Z.C.; Chen, H.W.; Mallikaratchy, P.; Sefah, K.; Yang, C.J.; Tan, W. Aptamers evolved from live cells as effective molecular probes for cancer study. Proc. Natl. Acad. Sci. USA. 2006, 103, 11838-11843.

36. Shangguan, D.; Cao, Z.C.; Li, Y.; Tan, W. Aptamers evolved from cultured cancer cells reveal molecular differences of cancer cells in patient samples. Clin. Chem. 2007, 53, 1153-1155.

37. Shangguan, D.; Tang, Z.; Mallikaratchy, P.; Xiao, Z; Tan, W. Optimization and modifications of aptamers selected from live cancer cell lines. Chembiochem. 2007, 8, 603-606.

38. Tang, Z.; Shanggunan, D.; Wang, K.; Shi, H.; Sefah, K.; Mallikratchy, P.; Chen, H.W.; Li, Y.; Tan, W. Selection of aptamers for molecular recognition and characterization of cancer cells. Anal. Chem. 2007, 79, 4900-4907.

39. Mukaratirwa, S.; de Witte, E.; van Ederen, A.M.; Nederbragt, H. Tenascin expression in relation to stromal tumour cells in canine gastrointestinal epithelial tumours. J. Comp. Pathol. 2003, 129, 137-146.

40. Cerchia, L.; Ducongé, F.; Pestourie, C.; Boulay, J.; Aissouni, Y.; Gombert, K.; Tavitian, B.; de Franciscis, V.; Libri, D. Neutralizing aptamers from whole-cell SELEX inhibit the RET receptor tyrosine kinase. PLoS. Biol. 2005, 3,e123.

41. Guo, K.T.; SchAfer, R.; Paul, A.; Gerber, A.; Ziemer, G.; Wendel, H.P. A new technique for the isolation and surface immobilization of mesenchymal stem cells from whole bone marrow using high-specific DNA aptamers. Stem Cells. 2006, 24, 2220-2231.

42. Guo, K.T.; Scharnweber, D.; Schwenzer, B.; Ziemer, G.; Wendel, H.P. The effect of electrochemical functionalization of Ti-alloy surfaces by aptamer-based capture molecules on cell adhesion. Biomaterials. 2007, 28, 468-474.

43. Khademhosseini, A.; Langer, R. Nanobiotechnology-Drug delivery and tissue engineering. Chemical Engineering Progress. 2006, 102, 38-42.

44. Smith, J.E.; Medley, C.D.; Tang, Z.; Shangguan, D.; Lofton, C.; Tan, W. Aptamer-conjugated nanoparticles for the collection and detection of multiple cancer cells. Anal. Chem. 2007, 79, 3075-3082.

45. Herr, J.K.; Smith, J.E.; Medley, C.D.; Shangguan, D.; Tan, W. Aptamer-conjugated nanoparticles for selective collection and detection of cancer cells. Anal. Chem. 2006, 78, 2918-2924.

46. Lee, J.O.; So, H.M.; Jeon, E.K. Aptamers as molecular recognition elements for electrical nanobiosensors. Anal. Bioanal. Chem. 2008, 390(4), 1023-1032.

47. Wang, W.; Chen, C.; Qian, M.; Zhao, X.S. Aptamer biosensor for protein detection using gold nanoparticles. Anal. Biochem. 2008, 373(2), 213-219.

48. Huang, C.C.; Chiu, S.H.; Huang, Y.F.; Chang, H.T. Aptamer-functionalized gold nanoparticles for turn-on light switch detection of platelet-derived growth factor. Anal. Chem. 2007, 79, 47984804. 
49. Choi, J.H.; Chen, K.H.; Strano, M.S. Aptamer-capped nanocrystal quantum dots: a new method for label-free protein detection. J. Am. Chem. Soc. 2006, 128, 15584-15585.

50. Natt, F. siRNAs in drug discovery: target validation and beyond. Curr. Opin. Mol. Ther. 2007, 9, 242-247.

51. de Fougerolles, A.; Vornlocher, H.P.; Maraganore, J.; Lieberman. Interfering with disease: a progress report on siRNA-based therapeutics. J. Nat. Rev. Drug Discov. 2007, 6, 443-453.

52. McNamara, JO.2nd.; Andrechek, E.R.; Wang, Y.; Viles, K.D.; Rempel, R.E.; Gilboa, E.; Sullenger, B.A.; Giangrande, P.H. Cell type-specific delivery of siRNAs with aptamer-siRNA chimeras. Nat. Biotechnol. 2006, 24, 1005-1015.

53. Chu, T.C.; Twu, K.Y.; Ellington, A.D.; Levy, M. Aptamer mediated siRNA delivery. Nucleic Acids Res. 2006, 34, e73.

C 2008 by MDPI (http://www.mdpi.org). Reproduction is permitted for noncommercial purposes. 